\title{
BLIND TO CHEMISTRY: MOLECULAR CONTAMINANT FILMS WE COULD BE MISSING DURING VISUAL INSPECTIONS AND THE POTENTIAL IMPACT TO SYSTEM PERFORMANCE
}

\author{
Dr. Elaine Seasly \\ NASA Langley Research Center \\ Walter Wrigglesworth III \\ Wyzkyds Consulting, LLC
}

\begin{abstract}
Throughout the assembly, integration and test process, molecular contamination levels of space mission hardware are monitored to meet system performance requirements. Qualitatively, reflective surfaces and witness mirrors are continuously inspected for the visible presence of molecular contaminant films. Quantitatively, periodic reflectance measurements of witness mirrors indicate changes of mirror reflectivity over time due to the accumulation of molecular contaminant films. However, both methods only consider the presence of a contaminant film and not the molecular composition. Additionally, there is a risk that hardware may appear to be "visibly clean" even with a molecular contaminant film present on critical surfaces. To address these issues, experiments were performed to quantify the maximum molecular contaminant film that could be missed in visual inspections on witness mirrors with five different contaminants present. The corresponding changes in mirror reflectivity were modeled using the program STACK to determine the impact to space mission hardware performance. The results of this study not only show the criticality in considering the chemical make-up of molecular contaminant films on system performance, but also the need to recognize and understand the limitations of traditional visual inspection techniques on detecting molecular contaminant films.
\end{abstract}

\section{INTRODUCTION}

Visual inspections are relied upon heavily throughout the space system hardware assembly, integration, and test (AI\&T) phase of the system lifecycle to check for quality and workmanship issues, verify completion of assembly processes, and to verify requirements. Visual inspections are relatively simple to perform, can be performed by multiple operators, can be repeated numerous times, and do not require contact with the hardware surface to perform the inspection. One requirement that is satisfied through visual inspections is the "visibly clean" requirement. "Visibly clean" means the hardware surface being inspected is free of any visual signs of particulate or molecular contamination. These visual inspections are performed under the lighting requirements and surface viewing distance conditions defined by IEST-STDCC1246E [1]. Typically, these inspections are performed with white light followed by ultraviolet light, as the different lighting sources can assist in detecting different contaminants. A visual inspection of a mirror segment of the James Webb Space Telescope is shown in Figure 1.

Presented at the $30^{\text {th }}$ Space Simulation Conference, Annapolis, MD, November 2018 


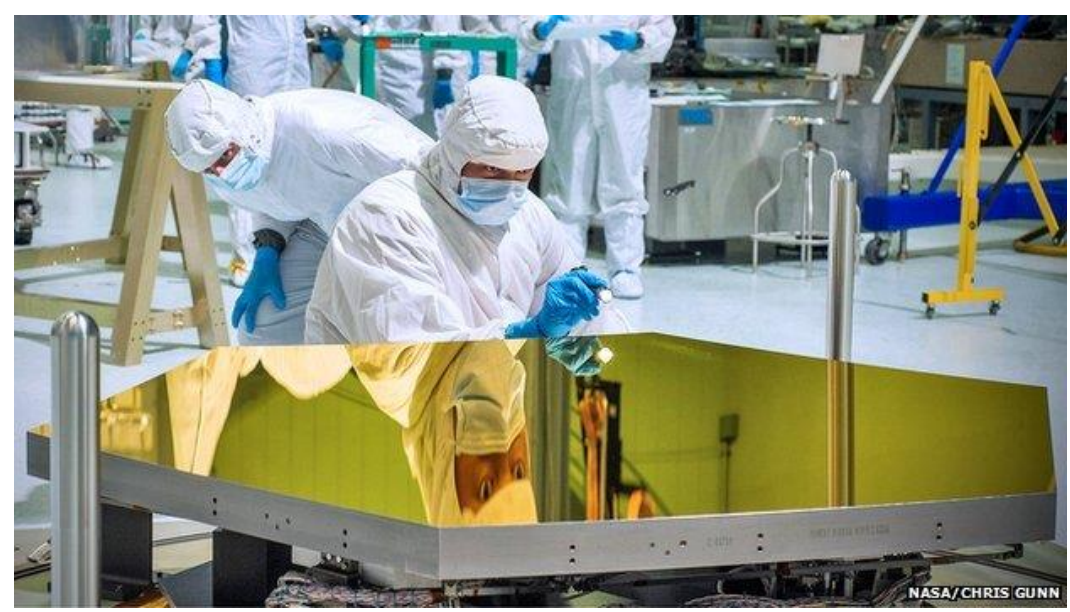

Figure 1: Visual Inspection of a James Webb Space Telescope Mirror Segment (NASA/Chris Gunn)

The ability to detect contaminants on surfaces depends on the contrast between the contaminant and the surface. As surface roughness increases, the ability to detect particles or molecular films on those surfaces decreases because the view of the contaminant can become "lost" in the background of the surface. Therefore, highly reflective surfaces such as mirrors are more desirable for visually detecting the presence of contaminants. Witness mirrors are utilized for monitoring contamination deposition during hardware AI\&T by being placed near the hardware during exposure to these environments. These mirrors are easily accessible, and can be continuously visually inspected for contamination. Additionally, witness mirrors can be optically characterized and periodically exchanged during the AI\&T process to measure the reflectance change over time.

Key decisions must be made if contaminants are detected through visual inspections or witness mirror reflectance measurements. Project decision makers must consider the information known about the contamination in the context of the complexity of the hardware built, and the project schedule and cost constraints. Unfortunately, chemical information on contamination cannot be obtained through visual inspections or reflectance measurements alone, and additional spectroscopic analysis is usually required to obtain this information. Analysis techniques such as gas chromatography-mass spectroscopy (GC-MS) or Fourier-transform infrared spectroscopy (FTIR) can provide chemical information on contaminants to help determine contaminant sources or mitigation techniques, but can require several days to perform and analyze results. In parallel, potential decisions such as cleaning the hardware to attempt to remove the contamination, reworking or replacing parts, or leaving the contamination in place and continuing forward in the project timeline are considered. The ability and potential cost to course-correct are greatly impacted by the complexity of the system and maturity of the hardware system through the AI\&T process. Hence, the goal for hardware visual inspections and analysis of witness plates is to find signs of contamination sooner rather than later, when coursecorrections are still possible and less costly to perform.

Despite widespread use as a standard, relatively little has been studied on the ability for observers to visually detect molecular contaminant films on surfaces at different film thicknesses. It is quite possible during the AI\&T phase for surfaces to be deemed visibly clean when a film thickness is below the visual detection limit, but present at a level that may be 
significant to cause system performance degradation. Chemical species present as molecular films on spacecraft surfaces can create system performance degradation effects such as reflection or transmission loss on optical surfaces and changes in emissivity of thermal control surfaces [2]. The presence of these films may not be detected until system performance is degraded when the system is exposed to space environments and operated on-orbit.

To begin to address these issues, experiments were performed to quantify the maximum contaminant film missed by observers performing visual inspections of different contaminant films on witness mirror surfaces. This metric was chosen as a worst-case scenario, as it provides project decision makers an idea of the film thickness that can go undetected by visual inspection techniques, and answers the question: "What could we be missing through our traditional visual inspection methods"? Experimental results were then modeled in a system performance degradation simulation to determine the effect molecular contaminant films had on changes in mirror reflectivity. By gaining a better understanding of the potential limitations of visual inspections in detecting different molecular contaminant films on witness mirror surfaces and the resulting impact to system performance, improved detection techniques, inspection processes, and system requirements may be developed.

\section{EXPERIMENTAL}

Five representative spacecraft molecular contaminant film materials were chosen for this study: silicone, hydrocarbon, fluorocarbon, ester, and a glycol polymer. Contaminants were chosen to represent different molecular chemistries from laboratory materials as shown in Table 1. Witness mirror substrates were $50.8 \mathrm{~mm}$ diameter $\mathrm{N}$-type phosphorous-doped single side polished silicon wafers purchased from University Wafer.

Table 1: Representative Spacecraft Molecular Contaminant Film Materials

\begin{tabular}{|l|l|}
\hline Sample Material & $\begin{array}{l}\text { Representative } \\
\text { Spacecraft } \\
\text { Contaminant }\end{array}$ \\
\hline Dow Corning® 704 diffusion pump fluid & Silicone \\
\hline $\begin{array}{l}\text { Kurt J. Lesker® Company KJLSS 15-19-20-70 } \\
\text { vacuum pump fluid }\end{array}$ & Hydrocarbon \\
\hline Miller-Stephenson MS-122AD release agent & Fluorocarbon \\
\hline $\begin{array}{l}\text { Loctite® } 242^{\mathrm{TM}} \text { Threadlocker methacrylate ester } \\
\text { sealant }\end{array}$ & Ester \\
\hline $\begin{array}{l}\text { Photonic Cleaning Technologies, LLC First } \\
\text { Contact }\end{array}$ & Glycol polymer \\
\hline
\end{tabular}

Requirements for molecular contamination films are expressed in terms of the concentration of nonvolatile residue (NVR) on surfaces in mass $/ 0.1 \mathrm{~m}^{2}$ per IEST-STD-CC1246E [1]. Contaminant solutions were gravimetrically prepared to IEST-STD-CC1246E NVR levels using an analytical balance with a precision and resolution accuracy of $0.1 \mathrm{mg}$. A solution of 1.0 $\mathrm{mg} / \mathrm{ml}$ was first prepared for each contaminant by adding the required mass of liquid 
contaminant by micro-pipette to a cleaned, dried, and pre-weighed glass bottle, adding the required amount of American Chemical Society (ACS) grade isopropyl alcohol (IPA) by mass, and thoroughly mixing the solution. IEST-STD-CC-1246E NVR Levels A through J are solution concentrations designated in terms of $\mathrm{mg} / 0.1 \mathrm{~L}$. For these levels, solutions were created by weighing out the necessary amount of $1.0 \mathrm{mg} / \mathrm{ml}$ solution and diluting with IPA to reach the concentration for the specified NVR level. NVR Levels A/2 to AA5 range from $\mu \mathrm{g} / 0.1 \mathrm{~L}$ to ng/0.1L, which required serial dilutions beginning with NVR Level A. For these levels, the required mass from the next higher level concentration of NVR solution were weighed and further diluted with the necessary amount of IPA.

Witness mirror substrates were weighed, deposited with a single droplet from the corresponding NVR solution bottle for the sample, and then weighed again. The diameter of the NVR droplet was measured with calipers after the droplet had completely spread over the surface but just prior to IPA evaporation as shown in Figure 2. Film thickness was calculated for each sample based on the concentration of the NVR solution, the diameter of the droplet, and assumed a solution density of $1 \mathrm{~g} / \mathrm{cm}^{3}$.

A sample was prepared for each of the 25 NVR levels of IEST-STD0CC1246E. Additionally, one blank (no NVR solution applied) and one control sample (100\% IPA solution) were created to account for any residues present in the IPA. Finally, samples were coded and randomized for the study. The 25 NVR levels, one blank, and one control sample created 27 samples for each contaminant. Multiplying by the five contaminants produced a total of 135 samples for the study. All sample preparations took place in an ISO Class 7 cleanroom environment to minimize environmental contamination contributions to the samples [3].

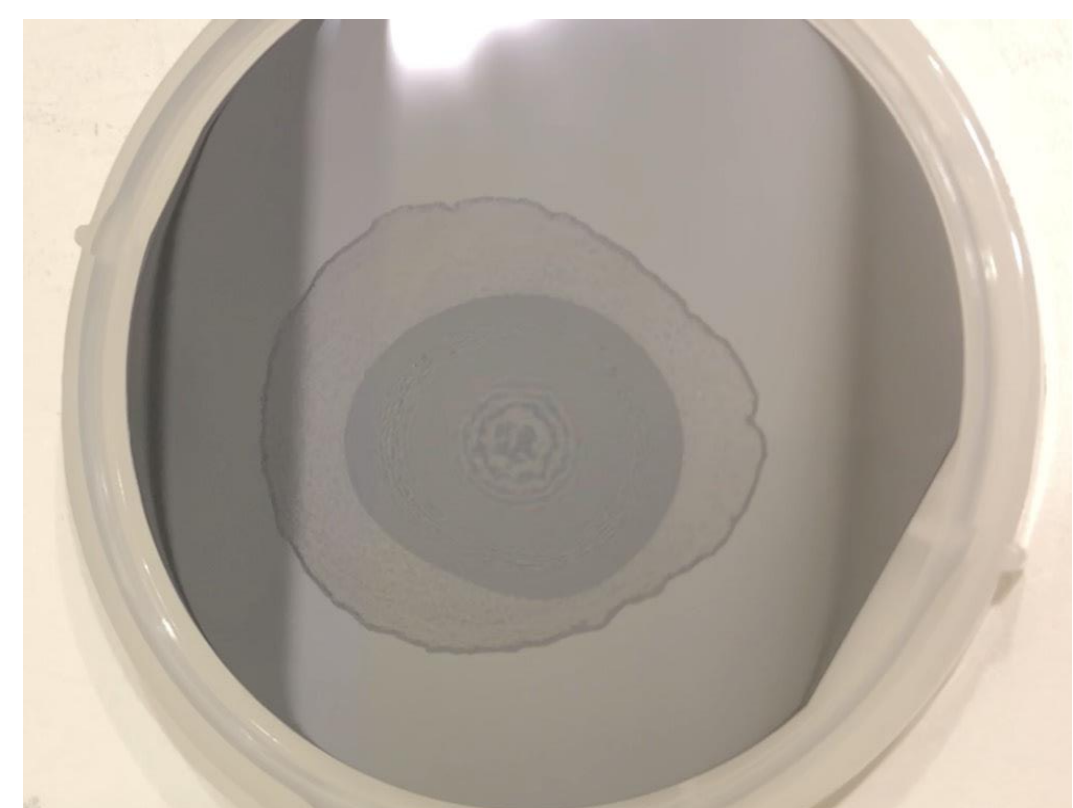

\section{Figure 2: Deposition of NVR droplet on Silicon Wafer Witness Mirror Substrate (NASA/Troy Gustke)}

VC-0.5-1000 inspection criterial from IEST-STD-CC1246E was used for this experiment. This criteria requires viewing samples at a distance of six to 18 inches and under a minimum of 100 foot-candle lighting. Randomized samples were placed face up in an identification grid on a cleanroom table in an ISO Class 7 cleanroom environment as shown in 
Figure 3. A close-up view of samples is shown in Figure 4. A vertical stand with lines delineating the required 6-18 inch viewing distance from the table surface was placed on the table with the samples. Ambient cleanroom lighting was used for illuminating samples, and a light meter measured the light intensity to verify the 100 foot-candle minimum lighting requirement was met.

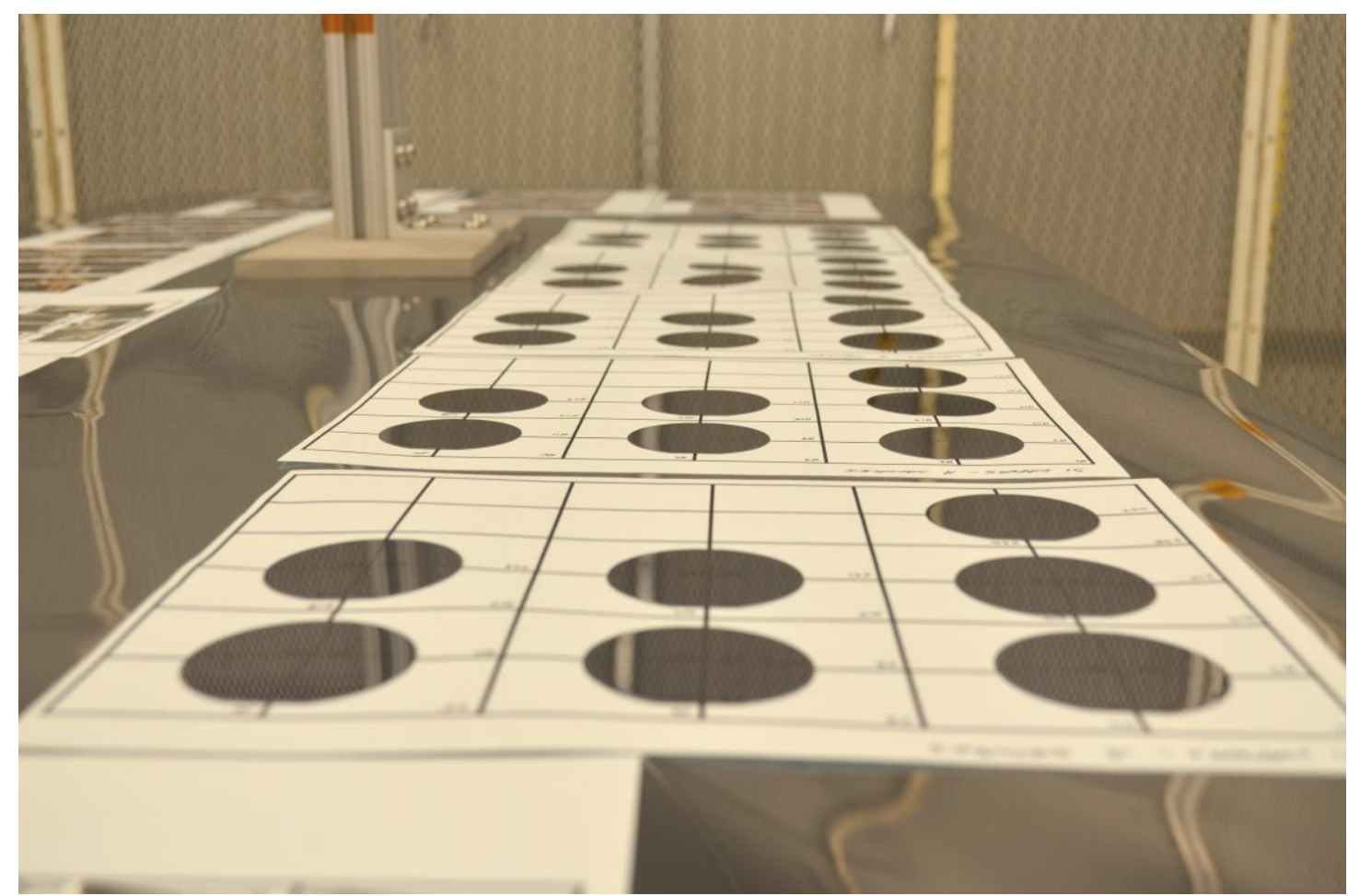

Figure 3: Randomized Witness Mirror Samples Arranged on a Cleanroom Table for Inspections (NASA/Joyce Corriere) 


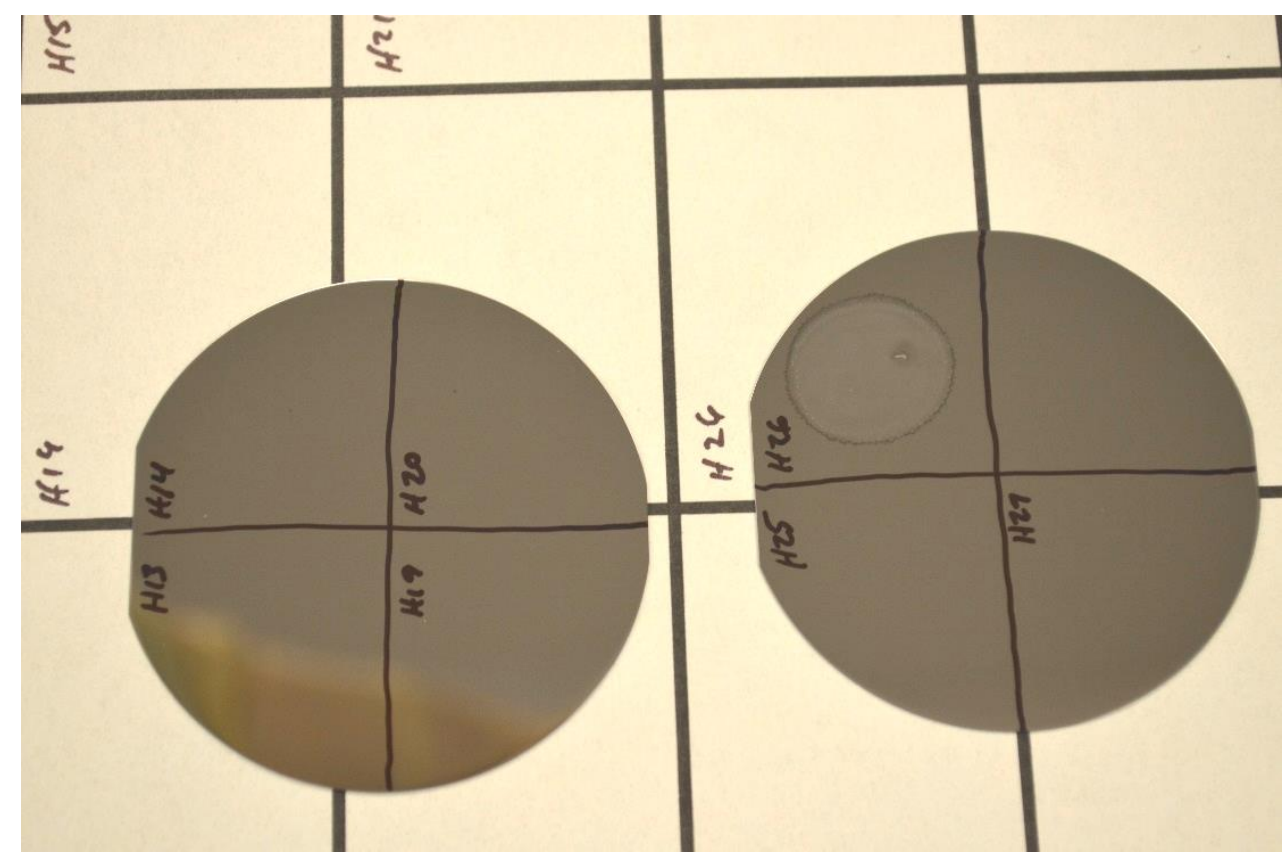

Figure 4: Close-up View of Contamination Samples (NASA/Joyce Corriere)

Five observers skilled in spacecraft integration and test at NASA Langley Research Center performed each visual inspection for this experiment. Each observer was instructed to review the IEST-STD-CC1246E inspection criteria, observe each sample with their eye level above the six inch mark but below the 18 inch mark on the vertical stand, and to observe surfaces as they would normally inspect spacecraft surfaces. No handling of the surfaces by the observer was allowed, however, observers could control their own viewing angle to the surface, much as they do during actual spacecraft visual inspections. Observers viewed surfaces with the unaided eye, but corrected vision was allowed. Each observer performed the visual inspections separately (no other observers in the room) and were instructed to identify if they detected any molecular film present with a Yes/No response. Data was recorded by the lead experimenter so that observers could focus on performing visual inspections without shifting their viewing gaze away from the samples to record results.

For each sample, a "Yes" response from an observer (positive detection) was recorded as a " 1 " and "No" response was recorded as a zero. The total positive detections for each sample were summed, and results were sorted in descending order. From this sorted table, the highest film thickness missed by any observer for each contaminant was flagged and recorded. For the purpose of this study, the worst-case film thickness missed was the metric of interest, regardless if one observer missed a positive detection of a sample or all five observers missed. Future studies and analysis will consider the probability distribution of positive sample detection amongst observers.

\section{PERFORMANCE MODELING}

The computer program STACK was used to model the reflectivity performance impact to mirrored spacecraft surfaces from incident light interacting with contaminant films on the surface. STACK was developed by the Arnold Engineering Development Center (AEDC) to model contaminant thin films in a "stack" on a defined substrate [4]. In this program, the user 
defines the substrate and contaminant layers, substrate thickness, contaminant film thicknesses, and inputs the optical constants (complex refractive index $n$ and $k$ values) for the substrate and contaminants over the spectral range of interest.

For this study, substrate film thicknesses were set to 25 micrometers in the model, which was 10 times greater than any film thickness analyzed. Model functionality was checked by modeling the reflectivity of polished bare aluminum as shown in Figure 5 [5]. Results matched published values from industry for all wavelengths from $300 \mathrm{~nm}$ to $2500 \mathrm{~nm}$ [6]. Reflection was modeled at a 90 degree angle of incident light to the substrate surface to simulate the highest energy impingement on the surface. For this case study, effects of only one contaminant on a substrate at a time were modeled. Future modeling efforts will model the effects of different contaminants stacked as layers on one another on a substrate.

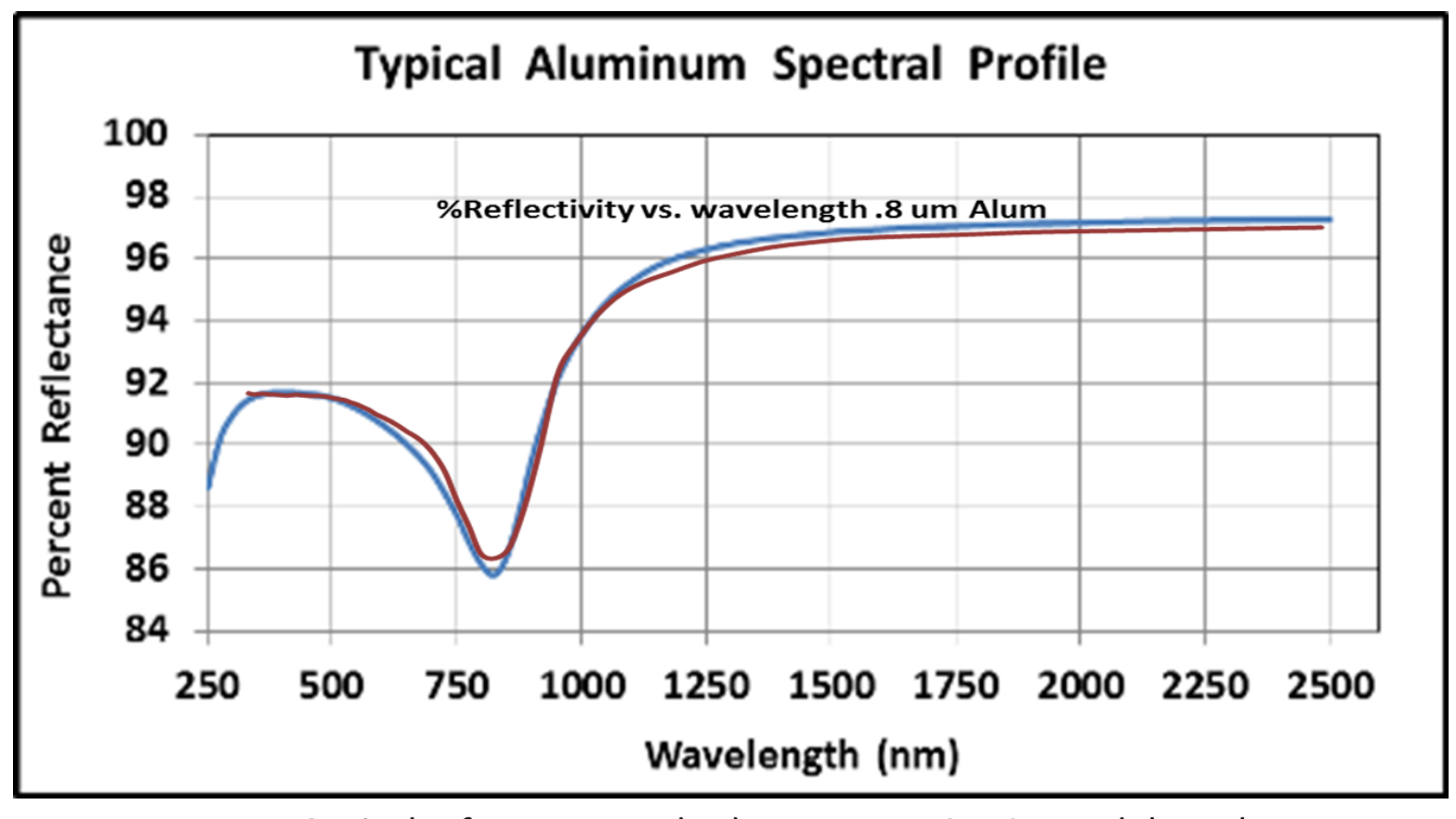

Optical reference standard

STACK model result

Figure 5: STACK Results for Bare Polished Aluminum Substrate as Compared to an Optical Reference Standard

\section{RESULTS \& DISCUSSION}

The maximum film thickness missed during visual inspections for each contaminant is shown in Table 2 [7]. The IEST-STD-CC1246E standard was used to set limits for this study, and higher values beyond this study are certainly possible. Reported values are not absolute for visual inspections, rather, they are relative based on the standard. Upon reviewing the results in Table 2 it is interesting to note that not all contaminants produced the same value for maximum film thickness missed by visual observers. Hydrocarbon was the contaminant with the lowest missed value at $1.74 \mathrm{~nm}$, while fluorocarbon had the highest film thickness missed at $22.25 \mathrm{~nm}$. 
Table 2: Maximum Contaminant Film Thickness Missed on Silicon Witness Mirrors During Visual Inspections

\begin{tabular}{|l|c|} 
Contaminant & $\begin{array}{c}\text { Maximum Film } \\
\text { Thickness Missed } \\
(\mathbf{n m})\end{array}$ \\
\hline Hydrocarbon & 1.74 \\
\hline Silicone & 2.31 \\
\hline Ester & 5.65 \\
\hline Glycol polymer & 18.91 \\
\hline Fluorocarbon & 22.25 \\
\hline
\end{tabular}

The performance modeling results of a molecular contaminant film present on a silicon mirror surface for the contaminants and film thicknesses listed in Table 2 are shown in Figure 6 [7]. Results are presented in terms of the percent change in reflectivity of the surface from 250 $\mathrm{nm}$ to $1450 \mathrm{~nm}$ when compared to a bare silicon mirror substrate with no contamination present. A typical requirement for silicon witness mirrors undergoing reflectivity measurements to monitor for contamination is no more than $1 \%$ change on the mirror throughout AI\&T. This maximum of $1 \%$ change in reflectivity is shown in Figure 6 in relation to the analysis model results. Most contaminants follow a similar curve shape and trend over the wavelength range. However, the fluorocarbon film produced a noticeably different effect from the other contaminants. A significant system performance impact could be realized if a $22.25 \mathrm{~nm}$ fluorocarbon film was missed during visual inspections, which is the level that occurred during the inspection experiments. The significance to performance impact depends on the operational wavelength region of interest. For example, in the visible wavelength range of $390 \mathrm{~nm}$ to 700 $\mathrm{nm}$, a $22.25 \mathrm{~nm}$ fluorocarbon film could cause a $0.7 \%$ to $1.9 \%$ change in silicon mirror reflectivity, respectively. The majority of this wavelength region would exceed the typical requirement of no more than $1 \%$ change in witness mirror reflectivity throughout the AI\&T phase. This result may be significant for the performance of systems that operate in this wavelength region, such as laser systems that operate at $488 \mathrm{~nm}$ or $532 \mathrm{~nm}$. 


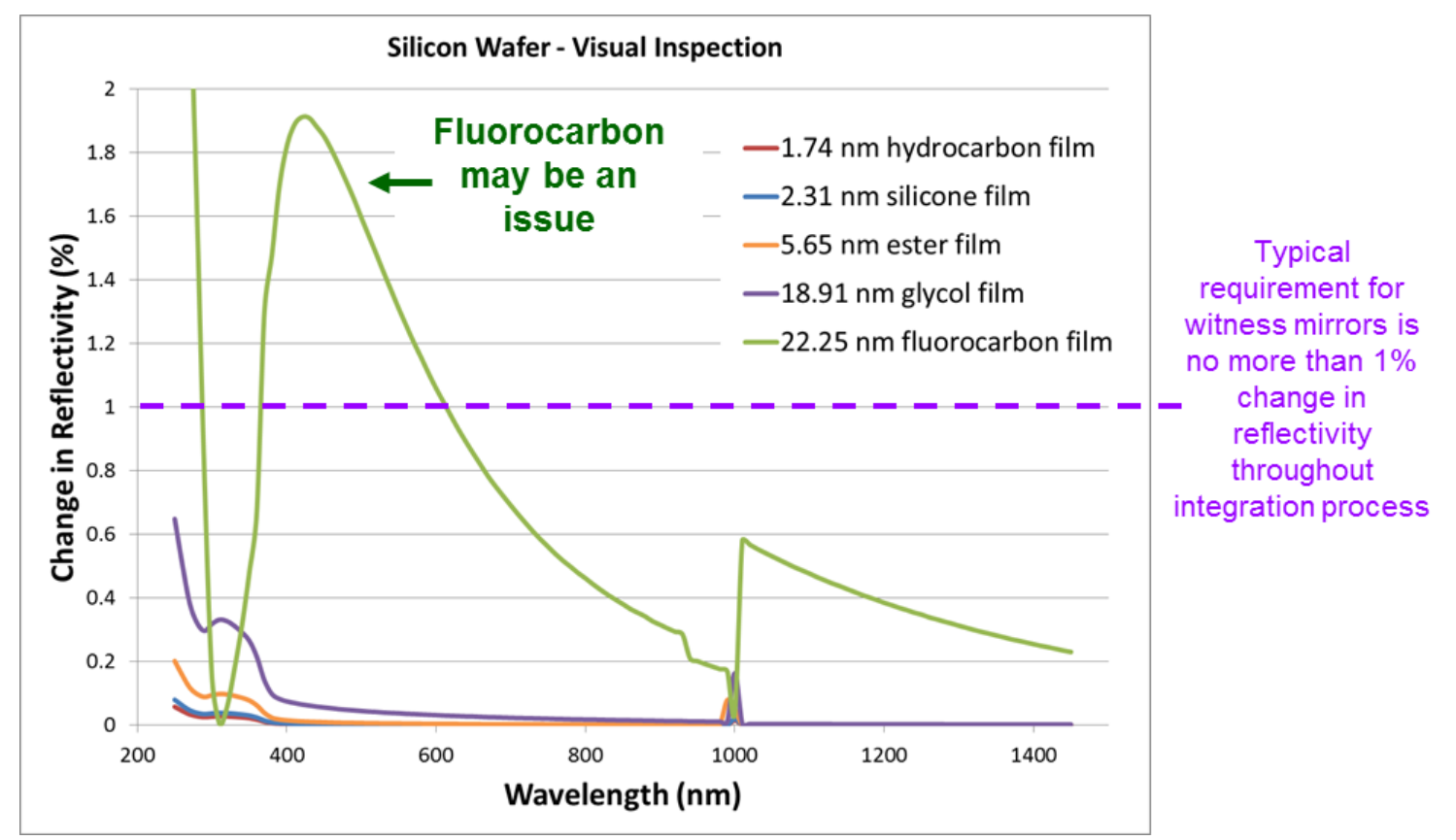

\section{Figure 6: Silicon Wafer Mirror Reflectivity Performance Impact Model Results for Visual Inspection}

The performance model results show the impact the presence of a contaminant film has on a space system surface such as a reflective mirror, and how a standard contamination detection method such as visual inspection can miss detecting a molecular contaminant film and the resulting system performance impact. As a result, this may impact how contamination control engineers set hardware cleanliness requirements and when inspections are performed. For contaminants that have a lower probability of visual detection, the contamination control engineer may need to increase the number of witness plates deployed and/or switch out the plates more frequently for analysis to quantitatively determine molecular contaminant film levels for contaminants of concern. Additionally, by knowing more about the limitations of visual inspections to different molecular contaminant film chemistries, better witness plate surfaces for visual inspections can be designed.

\section{CONCLUSIONS}

The goal for any contamination detection method is to miss detecting as low a film thickness of molecular contamination as possible. Previously, no empirical data existed on how well visual inspections detected molecular contaminant films on witness mirror surfaces, despite visual inspections being used as a standard. As a result of this study, the amount this method could miss in terms of maximum film thickness for different contaminants on different spacecraft surfaces has been determined, and the corresponding potential impact to space system performance modeled. In some cases, "visibly clean" may not be clean if a molecular film that cannot be visibly detected has built up to a level to impact system performance. The results of this study not only show the criticality in considering the chemical make-up of molecular contaminant films on system performance, but also the need to recognize and understand the limitations of traditional visual inspection techniques on detecting molecular contaminant films. 
This study has also shown that a contamination detection method such as visual inspection is not perfect, and any given method contains inherent limitations that can miss detecting molecular contaminant films. Characterizing and understanding these limitations is necessary for effectively utilizing contamination detection methods and developing future methods.

\section{ACKNOWLEDGEMENTS}

The authors would like to thank the reviewers and experimental observers in the Systems Integration and Test Branch at NASA Langlely Research Center for their support in this research.

\section{REFERENCES}

[1] IEST. IEST-STD-CC1246E: Product Cleanliness Levels - Applications, Requirements, and Determination 2013.

[2] Rampini R, Grizzaffi L, Lobascio C. Outgassing Kinetics Testing of Spacecraft Materials. Materwiss Werksttech 2003;34:359-64. doi:10.1002/mawe.200390075.

[3] ISO. ISO 14644-1: Cleanrooms and associated controlled environments - Part 1: Classification of air cleanliness by particle concentration 2015.

[4] Palmer KF, Williams MZ, Budde BA, Bertrand WT. Optical Analysis Methods for Material Films Condensed on Cryogenic Surfaces of Spacecraft. Tech. Rep. AEDC-TR94-3, AD-A284014, Defense Technical Information Center, Fort Belvoir, Va.; 1994.

[5] Seasly E, Wrigglesworth III W. Preventing Premature Death in the M \& S Lifecycle: Lessons Learned from Resurrection and Modernization of a Space System Contamination Model. ModSim World 2018, 2018.

[6] Optical Reference Laboratory Reflectance Standards n.d. http://opticalreferencelab.com/calibrated-specular-reflectance-standards/ (accessed May 8, 2017).

[7] Seasly E. Methodology to Evaluate Proposed Leading Indicators of Space System Performance Degradation Due to Contamination. The George Washington University, 2018. 